\title{
ASYLUM HOSPITALS.
}

The accompanying plate should have been bound up in the July number of the "Journal of Mental Science" as an illustration to Dr. Richard Greene's article on "Asylum Hospitals" (see July number, 1888, page 177). Owing to delay in delivery, the number was issued without it. 

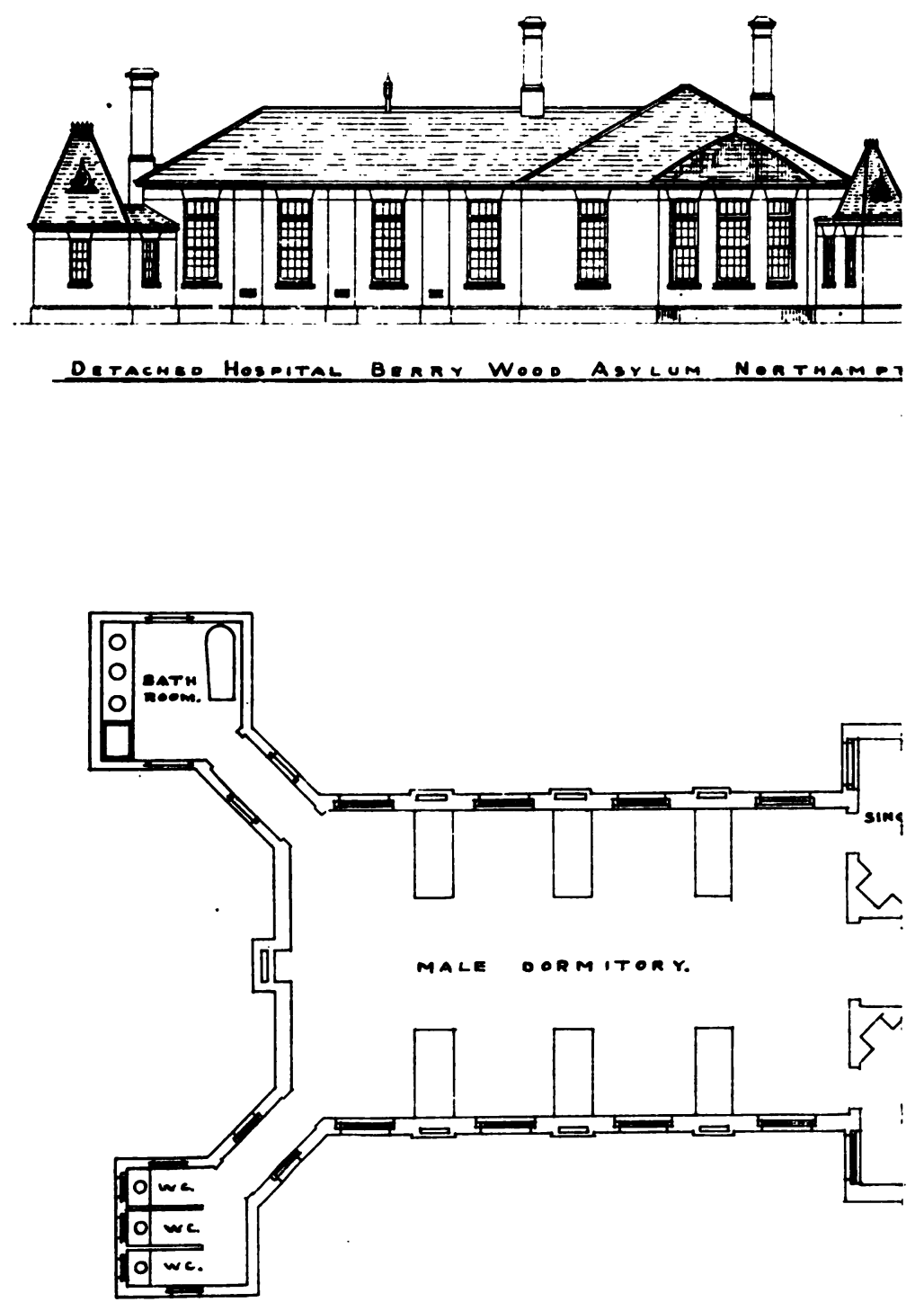
THE

\section{MEDICO-PSYCHOLOGICAL ASSOCIATION,}

THE COUNCIL, 1888-89.

PRESIDENT.-T. S. CLOUSTON, M.D., F.R.C.P.

PRESIDENT-ELECT.-H. F. HAYES NEWINGTON, M.R.C.P.

TrmasUrer.-JOHN H. PAUL, M.D.

EDITORS OF JOURNAI $\left\{\begin{array}{l}\text { D. HACK TUKE, M.D. } \\ \text { GEO. H. SAVAGE, M.D. }\end{array}\right.$

AUDITORS $\left\{\begin{array}{l}\text { D. YELLOWLEES, M.D. } \\ \text { T. OUTTERSON WOOD, M.D. }\end{array}\right.$

HON. SFCRETARY TOR IRRLAND.-CONOLLY NORMAN, F.R.C.S.I.

HON. BECRETART YOR SCOTLAND.-A. R. URQUHÄRT, M.D.

GENERAL SECRETARY.-HY. RAYNER, M.D.

H. T. PRINGLE, M.D.

G. E. SHUTTLEWORTH, M.D.

H. SUTHERLAND, M.D.

FLETCHER BEACH, M.D.

F. H. CRADDOCK, M.D.

F. SCHOFIELD, M.D.

8. W. D. WILLIAMS, M.D.

A. PATTON, M.B.

W. W. IRELAND, M.D.

W. J. DODDS, M.D.

S. A. K. STRAHAN, M.D.

E. B. WHITCOMBE, M.R.C.S.

F. M. $\mathrm{CO}(\mathrm{K}, \mathrm{M} . \mathrm{B}$.

E. M. COURTENAY, A.B., M.B.

T. D. GREENLEES, M.D.

M. D. MACLEOD, M.B.

J. WIGLESWORTH, M.D.

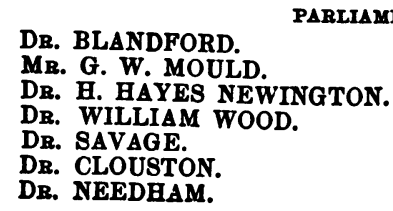

With power to add to their number.

DR. $\triangle D A M$.

DR. J. A. CAMPBELL.

DR. CA SSIDY.

DR. PRITCHARD DAVIES.

DR. GREEN

DR. MURRAY LINDSAY.

DR. BEVAN LEWIS.

DR. MERSON.

PENSIONS COMMITTEE.

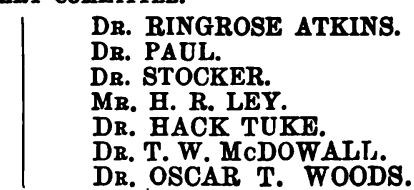

DR. RINGROSE ATKINS.

DR. PAUL.

DR. STOCKER.

DR, HACK TUKE

DR. T. W. McDOWALI.

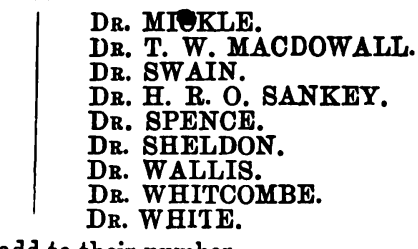

With power to add to their numben Members of the Association.

Adam, James, M.D. St. And., Private Asylum, West Malling, Kent. Adams, Josiah O., M.D. Durh., F.R.C.S.Eng., Brooke House, Upper Clapton,

$\Delta$ dams, Richard, L.R.C.P. Edin., M.R.C.S. Eng., Medical Superintendent, County Asylum, Bodmin, Cornwall.

Agar, S. H., L.K.Q.C.P., Hurst House, Henley-in-Arden.

Agar, S. Hollingsworth, jun., B.A. Cantab., M.R.C.S., Hurst House, Henlej-in. Arden.

Aitken, Thomas, M.D. Edin., Medical Superintendent, District Asylum, Inverness. Aldridge, Charles, M.D. Aberd., M.R.C.S., Plympton House, Plympton, Devon.

Alliott, A. J., M.D., St. John's, Sevenoaks. 
ii. Members of the Association.

Amsden, G. M.B., Medical Supt., County Asylum, Brentwood, Esser. Anderson, W. A.. M.B., Barming Heath Asylum.

Aplin, A., M.R.C.S.E. and L.R.C.P. Lond., Med. Supt. Co. Asylum, Bneaton,

Argo, G. C., M.B., Assist. Med. Officer, County Asylum, Sedgefield, Ferry Hill, Durham.

Armotrong, W., M.D., 16, Brunswick Street, Fitzroy, Melbourne, Victoris.

Ahe, Isaac, A.B., M.D., Medical Superintendent, Central Criminal Asylum, Dundrum, Ireland.

Atkins, Ringrose, M.A., M.D. Queen's Univ. Ire., Med. Superintendent, District Lunatic Asylum, Waterford.

Atkinson, R., B.A. Cantab., F.R.C.S., Sen. Assist. Med. Officer, Powick, near Worcester.

Baillarger, M., M.D., Member of the Academ y of Medicine, formerly Visiting Phygician to the Salpêtrière; 7, Rue de l'Université, Paris. (Hon.-Member.)

Baker, Begj. Ruseell, M.R.C.S. Eug., L.S.A., Assist. Med. Off, , Preetwich Aoylum Manchester.

Baker, H. Morton, M.B. Edin., Aseistant Medical Officer, Leicenter Borough Anylum. Leicester.

Baker, John, M.B., H.M. Convict Prison, Portsmouth.

Baker, Robert, M.D. Edin., Med. Supt., The Retreat, York.

Baker, John, 4, Anchorgate Terrace, Portsea.

Ball, Profeesor, Paris, Professor of Mental Diseases to the Faculty of Medicine, 179, Boulevard St. Germain, Paris. (Hon. Member.)

Banks, J. T., A.B., M.D. Trin. Coll. Dub. F.K. and Q.C.P. Ireland, Visiting Physician, Richmond District Asylum, 45, Merrion Square East, Dublin.

Banks, William, M.B. Lond., 3, Dunstanville Villas, Falmouth.

Barnes, J. F., 83, Caversham Street, London, N.W.

Barton, Jae. Edwd., L.R C.P. Edin., L.M., M.R.C.S., Medical Superintendent Surrey County Lunatio A sylum, Brookwood, Woking.

Bayley, J., M.R.C.S., Med. Supt., Lunatic Hospital, Northampton.

Beach, Fletcher, M.B., M.R.C.P. Lond., Medical Superintendent, Darenth Asylum, Dartford.

Benedikt, Prof. M., Franciskaner Platz 5, Vienna. (Hon. Member.)

Benham, H. A., M.B., C.M., As8. Med. Officer, City and County Asylum, Staple.

Biff, M., M., M.D., Editor of the Italian “" Journal of Mental Science," 16, Borgo di Ban Celso, Milan. (Honorary Member.)

Bigland, Thomas, M.R.C.S. Eng., L.S.A. Lond., Medical Superintendent, The Priory, Roohampton.

Blair, Robert, M.D., Ass. Med. Off. Woodilee Asylum, Lenzie, near Glasgow.

Blanchard, E. B., M.D., Medical Superintendent, Hospital for Insane, Charlotto Town Prince Ed ward's Island.

Blanche, M. lo Docteur, 15, Rue des Fontis, Autenil, Paris. (Hon. Member.)

Bland, W. C., M.R.C.S., Med. Supt., Borough Asylum, Portsmouth.

Blandford, George Fielding, M.D. Oxon., F.R.C.P. Lond., 71, Grosvenor Street, W. (PREsident, 1877.)

Blazland, Herbert, M.R.C.S. Med. Supt. Callan Park Asylum, New South Wales.

Bower, David, M.B. Aberd., Springfield House, Bedford.

Bowes, John Ireland, M.R.C.S. Eng., L.S.A., Medical Superintendent, County Asylum, Devires, Wilts.

Boys, A. H., L.R.C.P. Edin., Chequer Lawn, St. Albans.

Bramwell, Byrom, M.D., F.R.C.P.P. Ed., 23, Drumsheugh Gardens, Edinburgh.

Brayn, R., L.R.C.P. Lond., Invalid Convict Prison, Knapp Hill, Woking.

Brodie, David, M.D. St. And., L.R.C.S. Edin., 148, Brompton Road, London, S.W.

Brosins, Dr., Bendorf.Sayn, near Coblenz, Germany. (Hon. Member.)

Brown, John Ansell, M R.Ć.S. Eng., L.S.A. Lond., late Medical Staff, 18, Bassett Road, North Kensin ton, W

Browne, Sir J. Crichton, M.D. Edin., F.R.S.E., Lord Chancellor's Visitor, Now Law Courts, Strand, W.C. (Honorary Member.) (PResidere, 1878.)

Brown-Séquard, C., M.D., Faculté de Médicine, Paris. (Hon. Member.)

Brushtield, Dr., Budleigh Salterton, Devon.

Bru.hfield, Thomas, Jun., M D., Chartham Asylum, Kent.

Bryant, s. W., M.B. Ed., St. Peter, Tavey Rectury, Tavistock. 
Bucknill, John Charles, M.D. Lond., F.R.C.P. Lond., F R.S., J.P., late Lord Chancellor's Visitor; Hillmorton Hall, Rugby. (Editor of Journal, 1852-62.) (President, 1860.)

Burman, Wilkie J., M.D. Edin., Ramsbury, Hungerford, Berke.

Butler, J. S., M.D., late Nedical Superintendent of the Hartford Retreat, Hart. for ', Connecticut, U.S. (Hon. Member.)

Byas, Edward, M.R.C.S. Eng., 25, Belsize Park, Hampstead. N.W.

Cadell, Francis, M.D. Edin 5 Castle Terrace, Edinburgh.

Camer.n, R. W. D., M D., Midlothian and 'eebles District Asylum.

Campbell, Colin M., M.B., C.M., Medical Supt., Perth District Asylum, Murthly. Campell, John A., M.D.'Glas., Medical Superintendent, Cumberland and Westmorland Asylum, Garlands, Carlisle.

Campbell, P. E., M.B., C.M., Senior Assist. Medical Officer, District Asylum, Caterham.

Calmeil, M., M.D., Member of the Academy of Medicine, Paris, late Physician to the Asylum at Charenton, near Paris. (Honorary Member.)

Cameron, Sohn, M.B., C.M. Edin., Medical Supt., Argyll and Bute Agylum, Lochgilphead.

Case, H., M.R.C.S.. Med. Supt., Lea vesden, Herts .

Cassidy, D. M., M.D., C.M.McGill Coll., Montreal, D.Sc. (Pub. Health), Edin., F.R.C.S.Edin., Med. Superintendent, Connty Asylum, Lancaster.

Chambers, James, M.D. Ass, Med (1)f, (umberland and Westmorland Asylum.

Chapin, John B., M.D., Pennsylvania Hospital for the Insane, Philadelphia, U.S.A. (Hon. Member.)

Chapman, Thomas Algernon, M.D. Glas., L.R.C.S. Edin., Hereford Co. and City Asylum, Hereford.

Charcot, J. Mi., M.D., Physician to Balpetrière, 17, Quai Malaquais, Paris. (Hon. Member.)

Christie, Thomas B., M.D. St. And, F.R.S.E., F.R.C.P. Lond., F.R.C.P. Edin., Medical Superintendent, Royal India Lunatic Asylum, Ealing, W. (Hon. General Secretary, 1872.)

Christie, J. W. Stirling, M.D., Med. Supt., County Asylum, Stafford.

Clapham, Wm. Crochley S., M.D., M.R.C.P., The Grange, Rotherham.

Clapton, Edward, M.D. Lond., F.R.C.P. Lond., Physician, St. Thomas's Hospital, Visitor of Lunatics for Surrey; 10A, St. Thomas Street, Borougb.

Clark, Archibald C., M.D. Kdin., Medical Superintendent, Glasgow District

Asylum, Bothwell.
Clarke, Henry, L.R.C.P. Lond., H.M. Prison, Wakefield.

Cleaton, John D., M.R.C.S. Eng., Commissioner in Lunacy, 19, Whitehall Place. (Honorary Member.)

Clouston, T. S., M.D. Edin., F.R.C.P. Edin., F.R.S.E., Physician Superintendent, Royal Asylum, Morningside, Edinburgh. (Editor of Journal, 1873-1881.)

Cobbold, C. S. W., M.D., 29, Woodstock Road, Chiswick, W.

Collie, F. Lang, H.B. C.M., Montrose Royal Lunatic Asylum.

Collins, G. Fletcher, M.R.C.S.E., \&c.

Cones, John A., M.R.C.S., Burgess Hill, Sussex.

Compton, T. J., M.B., C.M. Aberd., Heigham Hall, Norwioh.

Cooke, Edwd." Marriott, M.B., M.R.C.S. Eng., Med. Supt., County Asylum, Worcester.

Cope, George P. L.K.Q.C.P.I. Richmond District Asylum, Ireland.

Courtenay, w. Maziere, A.B., M. B., C.M., T.C.D., Resident Physician-Superinten. dent, District Hospital for the Insane, Limerick, Ireland.

Cox, I. R., M.D., Med. Supt., County Asylum, Denbigh.

Craddook. F. H., B.A. Oxon, M.R.C.S. Eng., L.S.A., Med. Supt., County Aoylum, Wolton, Gloucester.

Curwen, J., M.D., Warren, Pennsylrania State Hospital for the Insane, U.S.A. (Hon. Member.)

Daniel, W. C., M.D. Heidelb., M.R.C.S. Eng., Epsom, Surrey.

Davidson, John H., M.D. Edinburgh, Medical Superintendent, County Asylum, Chester.

Davies, Francis P., M.B. Edin., M.R.C.S. Eng., Kent County Asylum, Barming Heath, near Maidstone.

Deas, Peter Maury, M.B. and M.S. Lond., Medical Superintendent, Wonford House, Exeter. 
Delaeiaure, M.. M.D., Member of the Academy of Medicine, Physician to the Bicétre, Paris, 35, Rue des Mathurins-Saint-Jacques, Paris. (Hon. Member.) Denholm, James, M.D., Duns, Berwickshire.

Denne, T. Vincent de, M.R.C.S. Eng., Colman Hill House, Halesowen, Worcester-

shire.
Déspine, Prosper, M.D., Rue du Loizir, Marseilles. (Honorary Member.)

Dickson, F. K., F.R.C.P. Edin., Wye House Lunatio Asylum, Buxton, Derbyehire. Dodds, Wm. J., M.D., D.Sc. Edin., Royal Asylum, Montrose.

Donaldson, R. Lockhart, M.B., District Asylum, Monaghan

Douty, J. Harriugton, Berks County Asylum, Moulsford, Wallingford.

Down, J. Langdon Haydon, M.D. Lond. F.R.C.P. Lond., late Resident Physician, Earlawood Asylum; 81, Harley St., Cavendish Sq., W., and Normansfield,

Hampton Wick.
Drapes, Thomas, M.B., Med. Supt., District Asylum, Enniscorthy, Ireland.

Dunlop, James, M.B., C.M., 423, St. Vincent Street, Glasgow.

Dwyer, J., L.R.C.P.I., Med. Supt., District Asylum, Cork, Ireland.

Eager, Reginald, M.D. Lond., M.R.C.S. Eng. Northwoods, near Bristol.

Eager, Wilson, L.R.C.P. Lond., M.R.C.S. Eng., Med. Superintendent, County Asylum, Melton, Suffolk.

Earle, Leslie, M.D. Edin, Melbourn Royston, Herts.

Earle, Pliny, M.D., Med. Superintendent, Northampton Hospital for the Insane, Mass., U.S. (Honorary Member.)

East, Edward, M.R.C.S. and L.S.A., 16, Upper Berkeley Street, W.

Eastwood, J. William, M.D. Edin., M. R.C.P. Lond., Dinsdale Park, Darlington.

Eoheverria, M. G., M.D., Havanns. (Honorary Member.)

Elliot, G. Stanley, M.R.C.P. Ed., L.R.C.S. Ed., Medical Superintendent, Caterham, Surrey.

Eustace, J., M.D. Trin. Coll., Dub., L.R.O.S.I.; Highfield, Drumcondra, Dublin.

Ewart, D. C. Theodore, M.B. Aberd., C.M., Leavesden Asylum, near Watford, Herts.

Falret, Jules, M.D., 114, Rue du Bac, Paris. (Honorary Member.)

Finch, W. Corbin, M.R.C.S. Eng., Fisherton Honse, Salisbury.

Finch, John E. M., M.B., Medical Superintendent, Borough Asylum, Leicester.

Finlayson, James, M.B., 351, Bath Crescent, Glasgow.

Finnegan, A. D. O'Connell, L.K. and Q.C.P.I., Med. Supt., District Asylum, Mullingar.

Fitzgerald, G. C., M.B., B.C. Cantab., Cane Hill Asylum, Surrey.

Fletcher, Robert Vicars, Esq., F.R.C.S.I., L.R.Q.C.P.I. and L.R.C.P. Ed., Medical Superintendent, District Asylum, Ballinasloe, Ireland.

Forrest, J. G. Stracey, L.R.C.P.Lond., M.R.C.S.Eng.

Fox, Charles H. M.D' St. And., M.R.C.S. Eng Brislington House, Bristol.

Fux, Bunville Bradley, M.A. Oxon., M.D., M.R.C.S., Brislington House, Bristol.

Francis, Lloyd, M.A., M.D. Oxon, St. Andrew's Hospital, Northampton.

Fraser, Donald, M.D., 44, High Street, Paisley.

Fraser, John., M.B., C.M., Deputy Commissioner in Lunaoy, 19, Stratham Road, Edinburgh.

Gairdner, W.T., M.D. Edin., Professor of Practice of Physic, 225, St. Vincent St., Glasgow. (PREsident, 1882.) (Honorary Member.)

Garner, W. H., Esq., F.R.C.S.I., A.B.T.C.D., Medical Superintendent, Clonmel District Asylum.

Gaequet, J. R., M.B. Lond., St. George's Retreat, Burgess Hill, and 127, Eastern Road, Brighton.

Gayton, F.C., M.D., Brookwood, Surrep.

Gelston, R. P., L.K. and Q.C.P.I., L.R.C.S.I., Modical Supt., District Asylum, Ennis.

Gill, Stanley A. M.R.C.P. Lond., M.R.C.S. Eng., Med. Superint., Rojal Lanatic Asylam, Liverpool.

Glendinning, James. M.D. Glas., L.R.C.S. Edin., L.M., Med. Supt., Joint Counties Asylum, Abergavenny.

Godding, Dr., Medical Superintendent Government Hospital for Insane, Washington, U.S (Honorary Member.)

Graham, W. S. M.B., Med. Supt., District Lunatic Asylum, Armagh.

Granville, J. M., M.D., Harewond Place, Hanover Square, London.

Greenlees, T. Duncan, M.D.. ' 'ity of London Iunutic Asylum, Stone, nnar Dartford.

Greene, Richard, L.R.C.P.Edin., Med. Superint., Berry Wood, near Northampton. 
Grubb, J. Strangman, L.R.C P. Ed., Silsoe Villa, Urbridgø Road, Ealing, W. Gwynn, 8. J., M.D., St. Mary's House, Whitechurch, Salop.

Habgood, W., L.R.C.P., Ass. Med. Uff.. Banstead Asylum, Surrey.

Hall, Ben., M.B.Lond., Assist. Med. Officer, The Brook Asylum, Liverpool.

Hall, Edward Thomas, M.R.C.S. Eng., Blacklands House Asylum, Chelsez.

Harbinson, Alexsnder, M.D. Irel., M.R.C.S. Eng., Assist. Med. Officer, County Asylum, Lancaster.

Harding, William, M.B., C.M. Ed., Assist. Med. Officer, County Asylum, Lancaster.

Harmer, Wm. Milsted, F.R.C.P. Ed., Physician Supt., North Grove House Asylum, Hawkhurst, Kent.

Harrey, Crosbie Bagnall, L.A.H., Asst. Med. Officer, District Asylum, Clonmel.

Hatchell, George W., M.D. Glas., L.K. and Q.C.P. Ireland, Inspector and Commise sioner of Control of Asylums, Ireland, 25, Upper Merrion Street, Dublin. (Hon. Member.)

Haughton, Rev. Professor S., School of Physio, Trinity Coll., Dublin, M.D., T.C.D., D.C.L. Oxon, F.R.S. (Hon. Member.)

Hearder, George J., M.D. St. And., L.R.C.S. Edin., Medical Superintendent, Joint Counties Asylum, Carmarthen.

Henley, E. W., L.R.C.P., County Asylum, Gloucester.

Hetherington, Charles, M. B., Med. Supt., Distriot Asylum, Derry, Ireland.

Hewson, R. W., L.R.C.P. Ed., Med. Supt., Coton Hill, Stafford.

Hicks, Henry, M.D., Hendon Grove House, Hendon.

Higgins, Wm. H., M.B., C.M., Med. Supt., County Asylum, Leicester.

Hill, Dr. H. Gardiner, Assist. Med. Officer, Cane Hill Asylum, Purley, Surrey.

Hills, William Charles, M.D. Aber., M.R.C.S. Eng., Thorpe, St. Andrew, near Norwich.

Hingston, J. Tregelles, Esq., M.R.C.S. Eng., Medical Superintendent, North Riding Asylum, Clifton, York. Hitchcock, Charles, L.R.C.P. Edin., M.R.C.S. Eng., Fiddington House, Market
Lavington, Wilts.

Hitchcock, Charles Knight, M.D., Bootham Asylum, York.

Hitchman, J., M.D. St. And. F.R.C.P. Lond., F.R.C.S. Fng. late Medical Superintendent, County Äsylum, Derby ; The Laurels, Fairford. (Prersidrent, 1856.)

Howden, James C., M.D. Edin., Medical Superintendent, Montrose Royal Lanatio Asylum, Sunnyside, Montrose.

Hughes, C. H., M.D., St. Louis, Missouri, United States. (Hon. Member.)

Humphry, John, M.R.C.S. Eng., Medical Superintendent, County Asjlum, Aylesbury, Bucks.

Hutson, E., M.D. Ed., Medical Superintendent, Lunatio Asylam, Barbadoes.

Hylop, Thos. B., M.B. Edin., Glasgow Distriot Asylum, Bothwell

Hyslop, Thos. B. M.B. Edin., Glasgow Distriot Asylum, Bothwell. Iles, Daniel, M.R.C.S. Eng., Resident Medical Officer, Fairford House Retreat,

Inglis, Thomas, F.R.C.P. Edin., Cornhill, Lincoln.

Ireland, W. W., M.D. Edin., Preston Lodge, Prestonpans, East Lothian.

Isaac, J. B., M.D. Queen's Univ., Irel., Assist. Med. Officer, Broadmoor, near Wokingham.

Jackson, J. Hughlings, M.D. St. And., F.R.C.P. Lond., Physician to the Hospital for Epilepsy and Paralysis, \&c.; 3, Manchester Square, London, W.

Jackson, J. J., M.R.C.S Eng., Cranbourne Hall, Grouville, Jersey.

Jamieson, Robert, M.D. Edin., L.R.C.S. Edin., Medical Superintendent, Royal Asylum, Aberdeen.

Jepson, Octavius, M.D. St. And., M.R.C.S. Eng., Conservative Club, London, S.W. Jeram, J. W., L.R.C.P., Hambledon, Cosham, Hants.

Johnston, D. G., M.B., C.M. Glas., Med. Supt., Moorcroft House, Hillingdon, Middlesex.

Johnston, J. A., L.R.C.S.I., Assist. Med. Officer, District Asylum, Monaghan, Ireland.

Johnstone, J. Carlyle, M.D., C.M., Medical Superintendent, Roxburgh, \&c., Dis trict Asylum, Melrose.

Jones, Evan, M.R.C.S. Eng., Ty-mawr, Aberdare, Glamorganshire.

Jones, D. Johnson, M.D. Edin., Senior Assistant Medical Officer, Kent County Asylum.

Jones, K., M.D. Lond., B.S., F.R.C.S., Earlswood Asylum, Surrey. 
A M RICAN A I TIUMg.

Demy 8vo. (with Portrait of Rusz), 78. 6d.

\section{The Insane in the United States and Canada,}

BY D. HACK TUKE, M.D., LL.D.

LONDON : H. K. LEWIS, 136, GOWER STREET, W.O.

By the Same Author.

I.-ILLUSTRATIONS OF THE INFLUENCE OF THE MIND

UPON THE BODY. 2nd Edit. 2 vols. 8vo, 15s.

J. AND A. Churchilt.

II.-INSANITY IN ANCIENT AND MODERN LIFE, WITH CHAPTERS ON ITS PREVENTION. 2nd Edition. $12 \mathrm{mo}, 6 \mathrm{~s}$.

Macmillan and Co.

III.-CHAPTERS IN THE HISTORY OF THE INSANE IN THE BRITISH ISLES. 8vo, $12 \mathrm{~s}$.

Krgan Paut, Trench, \& Co.

IV.-SLEEP-WALKING AND HYPNOTISM. 8vo, 58.

London: J. AND A. Churchill, 11, New Burlington Street.

\section{The Medico-Legal Journal,}

A Quarterly devoted to the Science of Medical Jurisprudence

(Published wnder the auspices of the Medico-Legal Society of the City of New York).

The price of the Medico-Legal Journal has been fixed at $\$ 3.00$ per annum, in the hope and with the expectation of its receiving a generous support from all classes of intelligent readers.

Subscriptions may be sent to Clarx Brel, Fesq., 57, Broadway, Now York.

\section{The American Journal of Insanity.} Published Quarterly at the State Lunatio Asylum, Utica, N. Y.

Subscription : Five dollars per annum.

Published monthly, 10 cts. per No., $\$ 1.00$ per year.

The International Record of Charities AND CORRECTION.

EDITED BY FREDERICK HOWARD WINES.

G. P. Putnay's Sons, New York and London.

\section{The Asclepiad :}

A Book of Original Research and Observation in the Science, Art, and Literature of Medicine, Preventive and Carative. Prioe 2s. 6d.

BY B. W. RICHARDSON, M.D., F.R.s. 
Kay, Walter S., M.B., Assistant Medical Ofiicer, South Yorkehire Asylum, Wadsley,

Keay, John, M.B., Med. Supt., Mavisbank, Polton, Midlothian.

Keegan, J. T., Indianapolis, Ind., U.S.A

Koch, Vincent, M.B., C.M., Borough Asylum, Cottingham, near Holl.

Kornfeld, Dr. Herman, Grottkau, Silesia, Germany. (Corresponding Member.)

Krafft-Ebing, R. v. M.D., Graz, Austria. (Hon. Mlemb.)

Laehr, H., M.D., Sohweizer Hof, bei Berlin, Editor of the "Zeitschrift für Psychia. trie." (Honorary Member.)

Lawrence, A., M.D., County Asylum, Chester.

Layton, Henry A., L. R.C.P. Edin. Cornwall County Asylum, Bodmin.

Legge, R. J., M.D., Assist. Med. Officer, County Asylum, Derby.

Leidesdorf, M., M.D. Universität, Vienna. (Honorary Member.)

Lentz, Dr., Asile d'Aliénés, Tournai, Belgique. (Hon. Member.)

Lewis, Henry, M.D. Bruss., M.R.C.S. Eng., L.S.A., late Assistant Modical Officer, County Asylum, Chester ; West Terrace, Folkestone, Kent.

Lewis, W. Bevau, L.R.C.P. Lond., Med. Supt., West Riding Asylum, Watefield.

Ley, H. Rooke, M.R.C.S. Eng., Medical Superintendent, County Asylum, Prestwich, near Manchester.

Lichfield, J. W., L.R.C.P. Lond., Assist. Med. Off., County Asylum, Fareham.

Lindsay, James Murray, M.D. St. And., F.R.C.S. and F.R.C.P. Edin., Med. Supt,, County Asylum, Mickleover, Derby.

Lisle, S. Ernest de, L.K.Q.C.P., Three Counties Asylums, Stotfold, Baldock.

Little, W. Maxwell, M.B. Edin., Assist. Med. Off., County Asylum, Thorpe, Norwich.

Lofthouse, Arthur, M.R.C.S., etc., Assist. Med. Off., County Asylum, Nottingham.

Lush, Wm. John Henry, F K.C.P. E'din., L.M., M.R.C.S. Eng., F.L.S., Fytield Honse, Andover, Hants.

Lyle, Thos., M.D. Glas., Rubery Hill Asylum, near Bromsgrove, Worcestershire.

MacBryan, Henry C. County Asylum, Hanwell, W.

MacDonald, P. W., II.D., C.M., Med. Supt., County Asylum, near Dorchester, Dorset.

Macfarlane, W. H., New Norfolk Asylum, Tasmania

Mackew, S., M.B. Edin., Hertford British Hospital, Rue de Villiers, LevalloisPerret, Seine.

Mackenzie, J. Cumming, M.B., C.M., County Asylum, Morpeth.

Mackintosh, Donald, M.D. Durham and Glas., L.F.P.S. Glas., 10, Lancaster Road, Belsize Park, N.W.

Maclaren, James, L.R.C.S.E., Stirling District Asylum, Larbert, N.B.

MacLean, Allan, L.R.C.S. Ed., Harpenden Hall, Herts.

Macleod, M. D., M.B., Med. Superintendent, East Riding Asylum, Beverley, Yorks.

Macphail, Dr. S. Rutherford, Derby Borough Asylum, Rowditch, Derby.

Madden-Medicott, Charles W. C., M.D. Edin., L.M. Edin., Ivy Dene, Teddington Park Road, Teddington.

Major, Herbert, M.D. 144, Manningham Lane, Bradford, Yorks, via Wakefield.

Manley, John, M.D. Edin., M.R.C.S. Eng., Denton House, Victoria Road, Southsea, Hants.

Manning, Frederick Norton, M.D. St. And., M.R.C.S. Eng., Inspector of Asylums for New South Wales Sydney. (Honorary Member.)

Manning, Harry, B.A. London, M.R.C.S., Laverstock House, Salisbury.

Manson, Magnus O., B.A., L.R C.P., etc., County Asylum, Haywards Heath, Sussex.

Marsh, James Welford, M.R.C.S. Eng., L.S.A., Assistant Medical Officer, County Asylum Lincoln

Marshall, William G., M.R.C.S., Medical Superintendent, County Asylum, ColneJ Hatch, Middlesez.

Mandsley, Henry, M.D. Lond., F.R.C.P. Lond., formerly Medical Superintendent, Royal Lunatic Hospital, Cheadle; 9, Hanover Square, London, W. (Editor of Journal. 1862.78.) (PBESIDENT, 1871.)

Maye, John, M.R.C.S. and L.S.A., Ass. Med. Off., Burntwood Asylum, Lichfield. McAlister, William, M.B., C.M., Struan Villas. Kilmarnock.

MoCreery, James Vernon, L.R.C.S.I., Medical Superintendent, New Lanatio Asylum, Melbourne, Australia.

McDonnell, Robert, M.D., T.C.D., F.R.C.S.I., M.R.I.A., Merrion Square, Dublin. 
McDowall, T. W., M.D. Edin., L.R.C.S.E., Medical Superintendent, Northumberland County Asylum, Morpeth. MoDowall, John Greig, M.B. Edin., Assist. Med. Officer, South Yorkshire Asylum,
Wadsley, Sheffield.

MoNaughtan, John, M.D., Med. Supt., Criminal Lunatic Asylum, Perth.

McPherson, John, M.B., Assistant Physician, Royal Edinburgh Asylum, Morn. ingside.

Merson, John. M.D. Aberd., Medical Superintendent, Borough Asylum, Hull.

Merrick, A.S., M.D. Qu. Uni. Irel., L.R.C.S. Edin., Medical Superintendent, District Asylum, Belfast, Ireland.

Meyer, Ludwig, M.D. University of Göttingen. (Honorary Member.)

Mickle, Wm. Julius, M.D., M.K.C.P., Med. Superintendent, Grove Hall Asylum, Bow, London.

Mickley, George, M.A., M.B. Cantab., Medical Superintendent, St. Luke's Hospital, Old Street, London, E.C.

Mierzejowski, Prof. J., Medico-Chirurgical Academy, St. Petersburg. (Hon. Member.

Miles, Geo. E., M.R.C.S., Callan Asylum, Sydney, N.S.W. Miller, Alfred, M.B. and B.C.L. Dub., Sen. Asst. Med. Officer, Hatton Asylum,

Mitchell, Sir Arthur, M.D. Aberd., LL.D., K.C.B., Commissioner in Lunacy for Scotland ; 34, Drummond Place, Edinburgh. (Honorary Member.)

Mitchell, R. B., M.D., Med. Supt. Midlothian District Asylum.

Mitchell, S., M.D. Edin., Medical Superintendent, South Yorkshire Asylum, Wadsley, near Sheffield.

Moloney, J. C., L.K.Q.C.P., Med. Supt., Patrick Hospital, Dublin.

Moody. James M., M.R.C.S. Eng., L.R.C.P. and L.M. Edin., Med. Supt., County Asylum, Cane Hill, Surrey.

Moore, E. E., M.B., Dub., Assist. Med. Officer, District Lunatic Asylum, Downpatrick, Ireland.

Moore, W. D., M.D., Assist. Med. Officer, Wilts County Asylum, Devizes.

Monro, Henry, M.D. Oxon, F.R.C.P. Lond., late Visiting Physician, St. Lake's Hospital ; 14, Upper Wimpole Street, London, W. (PRESIDENT, 1864.)

Morel, M.Jules, M.D., Hospice Guislain, Ghent. (Correspondzng Member.)

Mortimer, J. D., Assist. Med. Off., Milton Asylum, Portsmouth.

Motet, M., 161, Rue de Charonne, Paris. (Hm. Member.)

Mould, George W., M.R.C.S. Eng., Medical Superintendent, Royal Lunatio Hospital, Cheadle, Manchester. (PRESIDENT, 1880.)

Muirhead, Claud. M.D., F.R.C.P. Edin., 30, Charlotte Square, Edinburgh.

Mundy, Baron Jaromir, M.D. Würzburg, Professor of Military Hygiene, Oni. versität, Vienna. (Honorary Member.)

Murray, Henry G., L.K.Q.C.P. Irel., L.M., L.R.C.S.I., Assist. Med. Off., Prestwich Asslum. Manchester.

Myddelton-Gavey, E. H., M.R.C.S. and L.S.A., 64, St. Stephen's Street, Ipswich.

Myles, William Zachary, L.F.P.S., Med. Supt. District Lunatio Asylum, Kilkenny.

Needham, Frederick, M.D. St. And., M.R.C.P.Edin., M.R.C.S. Eng., late Medical Superintendent, Hospital for the Insane, Bootham, York ; Barnwood House, Gloucester. (PRESIDENT, 1887.)

Neil, James, M.D., Asst. Med. Officer, Warneford Asylum, Oxford.

Newington, Alexander, M.B. Camb., M.R.C.S. Eng., Woodlands, Ticehurst.

Newington, H. Hayes, M.R.C.P. Edin., M.R.C.S., Ticehurst, Sussex.

Newth, A. H., M.D., Haywards Heath, Sussex.

Nichols, C. H., M.D., Bloomingdale Asylum, New York. (Honorary Member).

Nicholson, William Norris, Esq., Lord Chancellor's Visitor of Lunatics, New Law Courts, Strand, W.C. (Honorary Member.)

Nicholson, W. R., M.R.C.S., Assistant Medical Officer, North Riding Asylum, Clifton, York

Nicolson, David, M.B. and C.M. Aber., late Med. Off., H.M. Convict Prison, Portse mouth. Med. Supt., State Asylum, Broadmoor, Wokingham, Berks.

Nielsen, Fred Wm., M.A. Cantab., M.R.C.S., \&c., County Asylum, Sedgfield, Ferry Hill, Durham.

Niven, William, M.D. St. And., Medical Staff H.M. Indian Army, late Superintendent of the Government Lunatic Asylum, Bombay, St. Margaret's, South Norwood Hill, S.E.

Nolan, Michael J., L.K.Q.C.P.I., Ass. Med. Off., Richmond Asylum, Dublin. 
viii.

Members of the Association.

North, S. W., Esq., M.R.C.S.E., F.G.S., 84, Micklegate, York, Visiting Medical Officer, The Retreat, York.

Norman, Conolly, F.R.C.S.I., Med. Supt., Richmond District Asylum, Dublin, Ireland. (Hon. Secretary for Ireland.)

Nugent, John, M.B. Trin. Col., Dub., L.R.C.S. Ireland, Senior Inspector and Commissioner of Control of Asylums, Ireland; 14, Rutland Square, Dublin. (Honorary Member.)

Oakshott, J. A., M.D., Assist. Med. Officer, District Asplum, Cork.

O'M eara, T. P., M.B., Med. Supt., District Asylum, Carlow, Ireland.

O'Neil, E. D., L.K.Q.C.P., Med. Supt., District Asylum, Castlebar.

Orange, William, M.D. Heidelberg, F.R.C.P. Lond., C.B., 35, Cromwell Road, Hove, Brighton. (PRESIDENT, 1883.) O'Shaughnessy, Thomas H., M.D., Ass. Med. Off., Ballinasloe District Asylum,
Ireland.

Owen, R. F., Tue Brook Villa, Liverpool.

Palmer, Edward, M.D. St. And., M.R.C.P. Lond., M.R.C.S., Medical Superintendent, County Asylum, Lincoln.

Parant, M. Victor, M.D., Toulouse. (Corresponding Member.)

Patton, Alex., M.B., Resident Medical Superintendent, Farnham House, Finglas, Co. Dublin.

Paul, John Hayball, M.D. St. And., M.R.C.P. Lond., F.R.C.P. Edin.; Camber. well House, Camberwell. (Treasurer.)

Peeters, M., M.D., Gheel, Belgium. (Hon. Member.)

Peddie, Alexander, M.D. Edin., F.R.C.P. Edin., F.R.S. Edin., 15, Rutland Street, Edinburgh.

Pedler, George H., L.R.C.P. Lond., M.R.C.S. Eng., 6, Trevor Terrace, Knightse bridge, $\mathbf{S}$. W

Petit, Joseph, L.R.C.S.I., Med. Supt., District Lunatic Asylum, Sligo.

Philipps, Sutherland Rees, M.D., C.M. Qu. Univ., Irel., F.R.G.S., St. Anne's Heath, Chertsey.

Philipson, George Hare, M.D. and M.A.Cantab., F.R.C.P. Lond., 7, Eldon Square,

Pewcastle.on-Tyne.

Pilkington, F. W., L.R.C.P. Lond., Ass. Med. Off., Littlemore, Oxford.

Pim, F., Esq., M.R.C.S. Eng., L.K. and Q.C.P. Ireland, Med. Supt., Palmerston, Chapelizod, Co. Dublin, Ireland.

Pitman, Sir Henry A., M.D. Cantab., F.R.C.P. Lond., 28, Gordon Square, W.C., Registrar of the Royal College of Physicians. (Honorary Member.)

Platt, Dr. St. James' Lodge, West End Lane, West Hsmpstend

Plaxton, Joseph Wm., M.R.C.S., L.S.A. Eng., Lunatic Asylum, Kingston, Jamaica.

Powell, Evan, M.R.C.S. Eng., L.S.A., Medical Superintendent, Borough Lunatic Asylum, Nottingham.

Powell, John, L.R.C.P., Senr. Asst. Med. Off., Joint Counties Asylum, Carmarthen.

Pringle, H. T., M.D. Glasg., Medical Superintendent, County Asylum, Bridgend, Glamorgan.

Rayner, Henry, M.D. Aberd., M.R.C.S. Eng., Medical Supt., County Asylum, Hanwell, Middlesex. (PRESIDENT, 1884.) (Honorary Gen. Secretary.)

Reid, William, M.D., Royal Asylum, Aberdeen.

Revington, Geo. M.B., Asst. Med. Off.. Prestwich Asylum, Manchester.

Reynolds, Graham H., M.B., C.M., Barnwood House, Gloucester.

Richardson, B. W., M.D. St. And., F.R.S., 25, Manchester Square, W. (Honorary Member.)

Robertson, Alexander, M.D. Edin., 16, Newton Terrace, Glasgow.

Robertson, Clarles A. Lockhart, ì.D. Cantab., F.R.C.P. Lond, F.R.C.P. Edin, Lord Chancellor's Visitor, Now Law Courts, Strand, W.C. (Generai Secretary, 1855-62.) (Editor of Journal, 1862-70.) (PRESIDENT, 1867.) (Honorary Member.)

Robertson, A. L. Fullarton, M.B., C.M.Ed., St. Andrew's, Billing Road, Northampton.

Robertson, G. M., M.B., C.M., Assistant Med. Off., Royal Asylum, Morningside, Edinburgh.

Rogers, Edward Coulton, M.R.C.S. Eng., L.S.A., Co. Asylum, Fulbourn, Cambridge.

Rogers, Thomas Lawes, M.D. St. And., M.R.C.P. Lond., M.R.C.S. Eng., Medical Superintendent, County Asylum, Rainhill, Lancashire. (President, 1874.) 
Ronaldson, J. B., L.R.C.P. Edin., Medical Officer, District Asplum, Haddington. Roots, William 8.. M.R.C.S., Onnhury House. Kingston-on-Thames.

Rorie, James, M.D. Edin., L.R.C.S. Edin., Modical Superintendent, Royal Asylum, Dundee. (Late Honorary Becretary for Scotland.)

Ross, Chisholm, M.B. Ed.. M.D. Sydney, Gladesville Asylum, New South Wales.

Roussel, M. Théophile, M.D., Sénateur, Paris. (Honorary Member.)

Rowe, E. L., I.R.C.P. Ed., Assist. Med. Oficer, Gloucester County Asylum.

Rowland, E. D., M.D., C.M. Edin., the Public Lanatio Asylum, Berbice, British Guiana.

Russell, A.P., M.B.Edin., The Lawn, Lincoln.

Russell, F. J. R., L.K.Q.C.P. Irel., 48, Lupus Street, London, W.

Rutherford, R. L., M.D., Medical Superintendent, City Asylum, Digby's, near Exeter.

Rutherford, James, M.D. Edin., F.R.C.P. Edin., F.F.P.S. Glasgow, Physician Superintendent, Crichton Rojal Institution, Dumfries. (Hon. Secretary for Scotland, 1876-86.

Rutherford, W. M.D., Consulting Physician, Ballinasloe District Asylum, Ireland. Sankey, H. R., M.B., Medical Superintendent, County Asylum, Hatton, Warwick. Sankey, B. Heurtley H., M.R.C.S. Eng., Medical Superintendent, Oxford County Asylum, Littlemore, Oxford.

Sankey, W. H. Octavius, M.D., F.R.C.P. Lond., Boreatton Park, near Shrewsbury, and Almund's Hotel, Clifton Street, Bond Street. (Presiders, 1868.)

Sarage, G. H.. M.D. Lond., Resident Physician, Bethlem Royal Hospital, London. (Editor of Journal.) (President, 1886.)

Schofield, Frank, M.D. St. And., M.R.C.S., Medical Supt., Camberwell House, Camberwell.

Schüle, Heinrich, M.D., Illenau, Baden, Germany. (Hon. Member.)

Scott, J. Walter, M.B.C.S., \&c., Assist. Med. Officer, County Asylum, Fareham, Hants.

Seccombe, Geo., L.R.C.P.L., The Colonial Lanatic Asylum, Port of Spain, Trinidad, West Indies

Seed, Wm., M.B., C.M. Edin., Assistant Modical Oficeer, Whittingham, Lancr-

Bells, H. T.. 2, London Road, Northfleet, Kent.

Semal, M., M.D., Mons, Belgium. (Hon. Member.)

Seward, W. J., M.D., Med. Superintendent, Colney Hatch, Middlesex.

Shaw, Thomas C., M.D. Lond., F.R.C.P. Lond., Medical Superintendent, Middlesex County Asylum. Banstead, Surrey.

Shaw, James, M.D., 63, Kensington, Liverpool.

Sheldon, T. S., M.B., Med. Supt., Cheshire County Asylum, Parkside, Macclesfield.

Sherrard, C. D., M.A.C.S., 17, The Arenue, Eastbourne.

Shuttleworth, G. E., M.D. Heidelberg, M.R.C.S. and L.S.A. Eng., B.A. Lond. Medical Superintendent. Royal Albert Asyluin, Lancaster.

Sibbald, John, M.D. Edin., F.R.C.P. Ed., M.R.C.S. Eng., Commissioner in Lanacy for Scotland, 3, St. Margaret's Road, Edinburgh. (Editor of Journal, 1871-72.) (Honorary Member.)

Sinclair, Eric, M.D. Med. Supt., Gladesville Asylum, New South Wales.

Skre, C. H., M.D. St. And., Medical Superintendent, Ayrshire District Asplum, Ayrshire, Glengall, Ayr.

Smith, Patrick, M.A. Aberdeen, M.D. Sydney, New Sonth Wales, Resident Med. Officer, Woogaroo Lunatic Asylum, Brisbane, Queensland, A ustralia.

Smith, Robert, M.D. Aberd., L.R.C.S. Edin., Medical Superintendent, County Asylum, Sedgefield, Durham.

Smith, R. Gillies, M.R.C.S., B.Sc. Lond., County Asylum, Sedgefield, Ferry Hill, Darham.

Smith, R. Percy, M.D., B.S., M.R.C.P., Bethlem Hospital, St. George's Road, S.E.

Smith, W. Beattie, F.R.C.S. Ed., L.R.C.P. Iond., Medical Supt., Hospital for the Insane, Ararat, Victoria.

Snell, Geo., M.R.C.S., Asst. Med. Off., Berbice, British Guians.

Soutar, J. G., Barnwood House. Gloucester.

Spence, James B., M.D. Ire. Med. Supt., Burntwood Asylum, Lichfield.

Spence, J. B., M.D., M.C., Q.U.I., Asylum for the Insane, Ceylon.

Spencer, Robert, M.R.C.S. Eng., Med. Superintendent, Kent County Asylum, Chartham, near Canterbury. 
Squire, R. H., B.A. Cantab., Assist. Medical Officer, Whittingham, Lancashire. Stearns, H. P., M.D., The Retreat, Hartford, Conn., U.S.A. Hm. Momber.)

Stewart, James, B.A. Queen's Univ., M.K.C.P. Edin., L.R.C.S. Ireland, late Assistant Medical Officer, Kent County Asylum, Maidstone; Duumurry, Sneyd Park, Clifton, Gloucestershire.

Stewart, Robert S., M.B., C.M., Assistant Medical Officer, County Asylum, Glanorgall.

Stewart, Rothsay C., M.R.C.S., Assist. Med. Officer, County Asylum, Leicester.

Stilwell, Henry, M.D. Edin., M.R.C.S. Eng., Mloorcroft House, Hillingdon, Middlesex.

Stocker, Alonzo Henry, M.D. St. And., M.R.C.P. Lond., M.R.C.S. Eng., Medical Superintendent, Peckham House Asylum, Peckham.

Stoker, Wm. Thornley, M.D., Consulting Physician, Swift's Hospital, 16, Harcourt Street, Dublin.

Strahan, S. A. K., M.D., Assist. Med. Officer, County Asylum, Berrywood, near Northampton.

Strange, Arthur, M.D. Edin., Medical Superintendent, Salop and Montgomery Asylum, Bicton, near Shrewsbury.

Street, C. T., M.R.C.S., L.R.C.P., Haydock Lodge, Ashton, Newton-le-Willows, Lancashire.

Suffern, A. C., M.D., Borough Asylum, Winson Green, Birmingham.

Sutherland, Henry, M.D. Oxon, M.R.C.P. London, 6, Richmoud 'Terrace, Whitehall, S.W.; Blacklands House, Chelsea; and Otto House, Hammersmith.

Sutton, H.' G., M.D. Lond., F.R.C.P., Physician to the London Hospital, 9, Finsbury Square, E.C

Swain, Edward, M.R.C.S., Medical Superintendent, Three Counties' Asylum, Stotfold, Baldock, Herts.

Bwanson, George J., M.D. Edin., Lawrence House, York.

Symes, G. D., M.R.C.S., County Asylum, Rainhill, Lancashire.

Tamburini, A., M.D., Reggio-Emilia, Italy. (Lon. Ifember.

Tate, William Barney, M.D. Aberd., M.R.C.P. Lond., M.R.C.S. Eng., Modical Superintendent of the Lunatic Hospital, The Coppice, Nottingham.

Thomas, C. G., M.B. Edin., Ass. Med. Off., CAterham Asylum, Surrey.

Thomson, D. G., M.D., C.M , Med. Supt., County Asylum, Thorpe, Norfolk.

Thurnam, Francis W yatt, M.B. Edin., C.M., 40, South Grove, Highgate, N.

Toller, Ebenezer, M.R.C.S. Eng., formerly Med. Supt. of St. Luke's Hospital, London, late Supt. of the Gloucester County Asylum, 10, Royal Crescent,

Holland Park, W.
Townsend, W. C., M.D., Visiting Physician, District Asylum, Cork.

Tuke, John Batty, M.D. Edin., 20, Charlotte Square, Edinburgh. (Honorary Secretary for Scotland, 1869-72.)

Tuke, John Batty, Junior, M.B., C.M., M.R.C.P.E., Resident Physician, Saughton Hall, Edinburgh

Tuke, Daniel Hack, M.D. Heidel., F.R.C.P. Lond., M.R.C.S. Eng., formerly Visiting Physician, The Retreat, York ; Lyndon Lodge, Hanwell, W., and 63, Wolbeck Street, W. (Editor of Journal.) (PaEstDenT, 1881.)

Take, Chas. Moulsworth, M.R.C.S., The Manor House, Chiswick.

Tuke, T. Seymour, M.R.C.S., Munor House, Chiswick.

Turnball, Adam Bobert, M.B., C.M. Edin., Medical Superintendent, Fife and Kinross District Asylum, Cupar.

Urquhart, Alexr. Reid, M.B., C.M., Physician Supt., James Murray's Royal Asylum, Perth. (Hon. Secretary for Scotland.)

Virchow, Prof. R., University, Berlin. (Hon. Member.)

Voisin, A., M.D., 16, Rue Séguin, Paris. (Hon. Member.)

Wade, Arthur Law, B.A., M.D. Dub., Med. Supt., County Asylum, Wells, Somerset. Walker, E. B. C., M.B., C.M. Edin., Assist. Med. Officer, County Asylum, Haywards Heath.

Wallace, James, M.D., Medical Officer, Parochial Asylum, Greenock.

Wallis, John A., M.B. Aberd., L.R.C.P. Edin., Medical Superintendent, County Asylum, Whittingham, Lancashire.

Walmsley, F. H., M.D., Leavesden Asylum.

Ward, Frederic H., M.R.C.S. Eng., L.S.A., Assistant Medical Officer, County Asylum, Tooting, Surrey.

Ward, J. Bywater, B.A., M.D. Cantab., M.R.C.S. Eng., Medical Superintendent, Warneford Asylum, Oxford. 
Warwick, John, F.R.C.S. Eng., 25, Woburn Square, W.C.

Watson, William Riddell, L. B.C.S. \& P. Edin., Govan Parochial Asylum, Glasgow. Weatherly, Lionel A., M.D., Bailbrook House, Bath.

Welsh, D., M. B., C.M., Assistant Medical Officer, Kent County Asylum, Barming Heath. West, Geo. Francis, L.R.C.P. Edin., Assist. Med. Officer, District Agylum, Omagh,
Ireland.

Westphal, C. Professor, Kronprinzenufer, Berlin. (Honorary Member.)

Whitcombe, Edmund Banks, Esq., M.R.C.S., Med. Supt., Winson Green Asylum,

White, Ernest, M.B. Lond., M.R.C.P., City of London Asylum, Stone, Dartford, Kent.

Wiokham, R. H. B., F.R.C.S. Edin., Medical Superintendent, Borough Lanatio Asylum, Newcestle-on-Tyne.

Wiglesworth, J., M.D. Lond., Rainhill Asylum, Lancashire.

Wilks, Samuel, M.D. Lond., F.B.C.P. Lond., Physician to Guy's Hospital ; 72, Grosvenor Street, Grosvenor Square.

Wilkes, James, F.R.C.S. Eng., late Commissioner in Lunacy; 18, Queen's Gardens, Hyde Park. (Honorary Member.)

Will, Jno. Kennedy, M.B., C.M., Bethnal House, Cambridge Road, E.

Willett, Edmund Sparshall, M.D. St. And., M.R.C.P. Lond., M.R.C.S. Eng Wyke House, Sion Hill, Isleworth, Middleser; and 4, Suffolk Place, Pall Mail.

Williams, S. W. Duckworth, M.D. St. And., L.R.C.P. Lond., 78, King's Roed, Brighton.

Williams, W. Rhys, M.D. St. And, M.R.C.P. Ed., F.K. and Q.C.P., Irel., Commissioner in Lunacy. 19, Whitehall Place. (Hon. Member).

Wilson, G. V., M.D., Assist. Med. Officer, District Asylum, Cork.

Wilson, Jno. H. Parker, H.M. Convict Prison, Brixton.

Winslow, Henry Forbes, M.D. Lond., M.R.C.P. Lond., 14, York place, Portman Square, London, and Hayes Park, Hayes, near Uxbridge, Middlesex.

Wood, William, M.D. St. And., F.R.C.P. Lond., F.R.C.S. Eng., Visiting Physician St. Lake s Hospital, formerly Medical Officer, Bethlem Hospital; 99, Harley Street, and The Priory, Roehampton. (President, 1865.)

Wood, Wm. E. R., M.A., M.B., F.R.C.S. Ėdin., Leighton House, Stanmore, Sydney, New South Wales.

Wood, Thomas Outterson, M.D., F.R.C.P., F.R.C.S. Edin., M.R.C.S. Engl., 40, Margaret Street, Cavendish Squari, W.

Wood, B. T., Esq., M.P., Chairman of the North Riding Asylum, Conyngham Hall, Knaresboro. (Honorary Member.)

Woods, Oscar T., M.B., M.D. (Dub.), L.R.C.S.I., Medical Superintendent, Asylum,

Woods, J. F., M.R.C.S., Med. Supt., Hoxton House, N.

Workman, J., M.D., Toronto, Canada. (Honorary Member.)

Worthington, Thos. Blair, M.A., M.B., and M.C. Trin. Coll., Dublin, Med. Supt., County Asylum, Knowle, Fareham, Hants.

Wright, Francis J., M.B. Aberd., M.K.C.S. Eng., Northumberland House, Stoke Newington, $N$

Wyatt, Sir William H., J.P., Chairman of Committee, County Asylum, Colney Hatch, 88, Regent's Park Road. (Honorary Member.)

Yellowlees, David, M.D. Edin., F.F.P.S. Glasg., Physician-Superintendent, Royal Asylum, Gartnavel, Glasgow.

Young, W. M., M.D., Assist. Med. Officer, County Asylum, Melton, Suffolk.

Younger, E. G., M.D. Bruss., M.R.C.P. Lond., M.R.C.S. Eng., Asst. Medical Officer, County Asylum, Hanwell, Middleser.

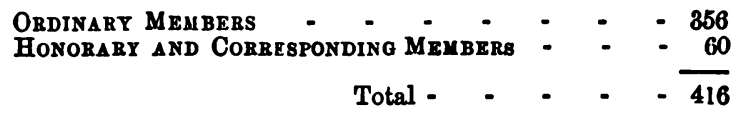

Members are particularly requested to send changes of address, \&c., to Dr. Rayner, the Honorary Secretary, County Asylum, Hanucll, Middlesex, and in duplicate to the Printer of the Journai, H.W.Wolfi, Lewes, Sussex. 
xii.

List of those who hate PASged the Examination FOR the Cemtificate of Efficiency in Paychological Medicine, entithing them to append M.P.C. (Med. Psych. Certif.)

TO THEIR NAMFS.

Armour, E. F.

Barker, Alfred James Glanville.

Blaok, Victor.

Bruco, John.

Calvert, William Dobreo.

Chapman, H. C.

Collie, Frank Lang.

Cope, George Patrick.

Cowper, John.

Cram, John.

Inglish, Edgar.

Erans, P. C.

Gemmell, William.

Fraser, Thomas.

Goodhall, Edwin.

Howden, Robert.

Hyslop, Thomas B.

Laing, J. H. W.

Leoper, Richard R.

Macevoy, Henry John.

Macpherson, John.

Melville, Henry B.

- Mortimer, John Desmond Frrnest.

Nairn, Robert.

Neil, James.

Nolan, Michael James.

Pearce, Walter.

Rice, P. J.

Rigden, Alan.

Robertson, G. M.

Rowland, Andrew.

Scott, J. Walter.

Staveloy, William Henry Charles.

Stoel, John.

Simpson, Samuel.

Slater, William Arnison.

Smith, Percy.

Symes, G. D.

Thompson, George Matthew.

Thorpe, Arnold $\mathbf{E}$.

Turner, M. A.

Waterston, Jane Elizabeth.

Will, John Kennedy.

Wilson, G. R.

Wood, David Jamer.

- To whom the Gaskell Prize (1887) was awarded. 
Second Brdition with coloured plates, 8vo, 12s. 6d.

\section{Lectures on Mental Disease.}

By W. H. O. SANKEY, M.D.LoND., F.R.C.P.,

Late Lecturer on Mental Disease University College, London.

The book bears evidence of great independence and originality of thought. The authore views are stated consisely and forcibly. It constitutes an important addition to peychological views are stated consisely and forcibly. It constitutes an important

London: H. K. LrwIs, 136, Gower Street.

Now ready. The Second Edition of

\section{FAGGE'S MEDICINE. \\ Revised and Fdited}

By DR. P. H. PYE-SMITH, F.R.S., Physician to Guy's Hospital.

N.B.-In this edition the Section on Insanity has been sapplied by Dr. G. H. SAVAG R.

Two volumes, 8vo, 38s., cloth ; 448., half.Persian.

London: J. \& A. ChurceilL, 11, New Barlington Street.

By W. R. Gowrzs, M.D., F.H.S.

Manual of Diseases of the Nervous System.

Vol. 1.-Diseases of the Spinal Cord and Nerves, with 171 Engravings (many figares). 8ro., 12s. 6d.

Vol. II.-Diseases of the Brain and Cranial Nerres; General and Functional Diseases, with 170 Engravings. 8vo., 178. 6d.

London: J. \& A. Churchill, 11, New Burlington Street.

\section{Fourth Edition, 12 Plates (4 being Coloured), 8vo, 258. \\ Manual of Psychological Medicine.} Br

J. C. BUCKNILL, M.D., F.R.C.P., F.R.S., and D. HACK TUKE, M.D., F.R.C.P

"It is not too much to say that it is the best and most favourably known to the medical and legal professions, by both of whom it is quoted as the higbest authority. It has acquired this enviable standing from the bigh repatation of it authors, from the correctness of the views and opinions expressed, and from its fulness. More than any work by English authors it deals with the subject of insanity in all its relations."-Amer. Journ. of Insanity.

London: J. \& A. Crurchild, 11, Now Burlington Street.

\section{Post 8vo, 6s.}

On some of the Mental Affections of Childhood

AND YOUTH, being the Lettsomian Lectures of 1887; together with Other Papers.

Bx J. LANGDON DOWN, M.D. Lond., F.R.C.P., Senior Physician to, and Lecturer on Clinical Medicine at, the London Hospital. "The most trustworthy work on this important and interesting subject."Lancet.

London: J. \& A. Churchild, 11, New Burlineton Street.

ON Aphasia : being a Contribution to the Subject of the Dissolution of Speech from Cerebral Disease. By JAMES ROSS, M.D., F.R.C.P., Senior Assistant Physician to the Royal Infirmary, Manchester.

J. \& A. Churchile, 11, New Burlington Street. 


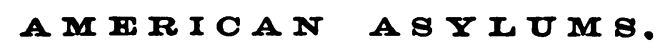

Demy 8vo. (with Portrait of Rusz), 78. 6d.

The Insane in the United States and Canada,

BY D. HACK TOKE, M.D., LL.D.

LONDON : H. K. LEWIS, 136, GOWER STREET, W.C.

\author{
By the Same Author. \\ I.-ILLUSTRATIONS OF THE INFLUENCE OF THE MIND \\ UPON THE BODY. 2nd Edit. 2 vols. 8vo, $15 \mathrm{~s}$. \\ J. AND A. Churchirt. \\ II.-INSANITY IN ANCIENT AND MODERN LIFE, WITH \\ CHAPTERS ON ITS PREVENTION. 2nd Edition. \\ $12 \mathrm{mo}, 68$. \\ Macuilean and Co. \\ III.-CHAPTERS IN THE HISTORY OF THE INSANE \\ IN THE BRITISH ISLES. 8vo, 128. \\ kequa Paul, Trench, \& Co. \\ IV.-SLEEP-WALKING AND HYPNOTISM. 8vo, $5 \mathrm{~s}$. \\ London: J. AND A. ChurchiLl, 11, New Burlington Street.
}

\title{
The Medico-Legal Journal,
}

A Quarterly devoted to the Science of Medical Jurisprudence (Published under the auspices of the Medico-Legal Society of the City of New York).

The price of the Medico-Legal Journal has been fixed at $\$ 3.00$ per annum, in the bope and with the expectation of its receiving a generous support from all classes of intelligent readers.

Subscriptions may be sent to Crarx Beld, Krsq., 57, Broadway, New York.

\section{The American Journal of Insanity. Published Quarterly at the State Lunatio Asylum, Utica, N. Y.}

Subscription : Five dollars per annum.

Published monthly, 10 cts. per No., 81.00 per year.

The International Record of Charities

AND CORRECTION.

EDITED BY FREDERICK HOWARD WINES.

G. P. Putnam's Sons, New York and London.

\section{The Asclepiad:}

A Book of Original Research and Observation in the Science, Art, and Literature of Medicine, Preventive and Curative. Price 2s. 6d.

BY B. W. RICHARD8ON, M.D., F.R.8.

Pablished Quarterly by

Lonoxans, Grems \& Co., Paternoster Row, London. 
COITHATS OF IO. 145.-APRT, 1888.

PART I. ORIGINAL ARTICLES.

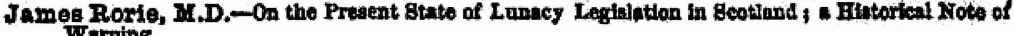

Rev. W. G. Devies, B.D.-The True Theory of Induction.

G. T. Revington, M.D. -The Neuroputhic Diatheals, or the Diatheals of the Degenerate.

George M. Robertson, M.B.-Reflex Speech.

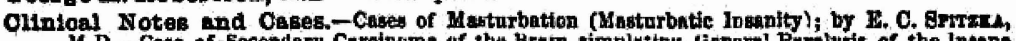
A.D. - Case of Seconiary Carcinums of the Bram gimblating Genernl Paralysis of the lineane

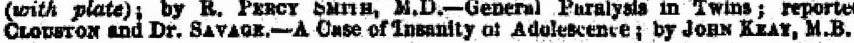

Oocasional Notes of the Quarter. -The Buser County Asylum Appointment,-Judiein Eccentricities.-Launcy Aets Amendment BAl.-Weik-minded Cinldren.

PART II.-REVIEWS.

The Nerrous Syutem and the Mind; a Treatise on the Dynamics of the Human Organtom; by $C$.

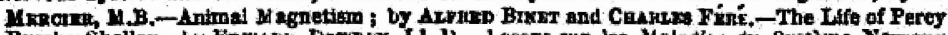

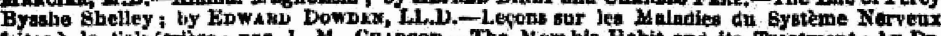
tites a la Swlpetrlère; par J. M. CaAncor. The Mort his Bubit and its Treatment; by Dr.

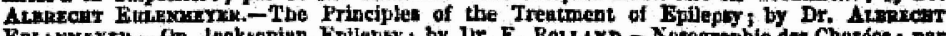

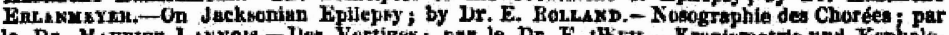

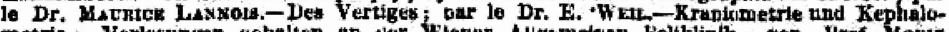
metrie; Vorlesungen Hehalten an Jer Wrener Al!kemetrien Poliklinlk; Van. Prot. Momiz BEN IDIET. - Ednchtional Kinds, or the Idend of Personal Developnient; by Somin BATANT, D.Se.

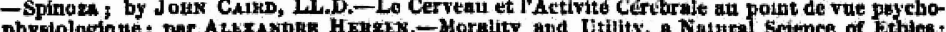
physlologique; par Alsx.

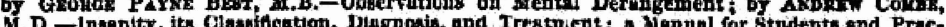

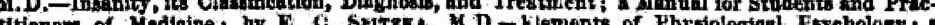
titioners of Treatioe on thie Activities and Nature of the ifind from the Physical and Ixperimental Potnt of

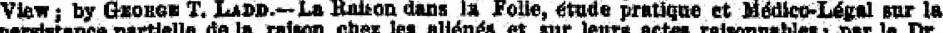
persiatance partiello

PART III.-PSYCHOLOGIOAL RETROSPEOT.

1. Rosian Retroepect. 2. Italian Retrospect; by J. R. GAseorer, M.B.

PART IV.-NOTES AND NEWS.

Quarteriy Meeting of the Medico-Paychological Association, heid at Bethlem Hospital on Friday, Feb. 24th.-Pas Examinations, - Special Meeting of the Metico-Psychnlogical Association, held to Bethlem Hcspital on Feb. 24th.-Quarterly Meeting of the scottish Branch of the MedicoAppolntmente.-Index Medico-Poychologicus.-Index to Vol, xixiti., do.

CONTHATS OF To. 146. -JUIY, 1888.

PART I.-ORIGINAL ARTICLES.

Fener Mandsley, M.D.,-Memarka on Crime and Crimimin.

G. T. Revington, M.D.-The Neuropathic Diathesis, or the Diatheals of the Degenerate.

Atohard Greene, L.R.C.P.-Arglum Hospitalk, with Pinus.

John Baker, M.B.-Bome Remarks on the Relation of Bpilepsy and Crime.

D. Feck Tuke, F.R.O.P.-On the Varlous Mlodes of Providing for the Insane and Iliots in the United Staces and Great Brituin.

Olinical Notes and Cases,- Recovery trom Chronle Insantty : Four Cusea ; by 8. A. K. Btunux, A.D. A Strange Case of Sulejue ; by W. B. TATE, M.D.-Cases of Masturbation (Masturbutic Insenity); by $\mathrm{F}$ C. SpITzkA, M.D.-General Paralysis of the Insane vimulating Corelsral Tumour; by G. H. SAYAGE, M.b.

Oocastonal Notes of the Quarter.-Trtal of Mason $v$. Marahall, Sunw, and Gunchard at the Oaficers,-Lunacy Acts Amendment Bill. The Provision for Idlots and Imbecties.

PART II,-REVIEWS.

A Mannal of the Disesses of the Nervous System; by W. R. Gowkm, H.D.-The Life of Percy Byshe

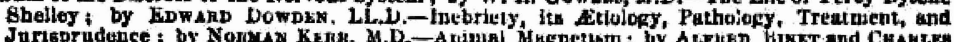

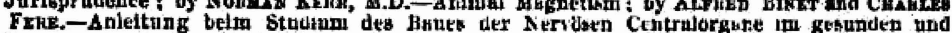

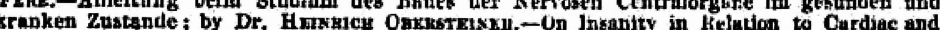

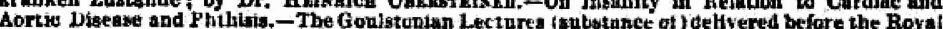

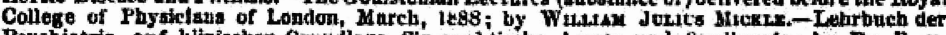
Psychintric, auf kjinisclier Grundlage flir praktische Aerzte und studiruvde; by Dr. R.

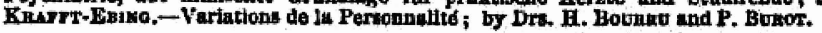

PART III.-PSYCHOLOGICAL RETROSPECT.

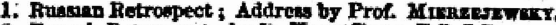

2. Fremeb Retrowpet; by D. HACr Tuts, F.R.C.P.

3. German Retrospect: by W. W. Iremaxi, M.D.

4. Inglitio Retrospect.

PART IV.-NOTES AND NEWS.

Qparterly Meeting of the Medico-Pejchological Associntion, held ot Bethlem Hospital-Speclal Meetíng on Parliamentary Billa,-Irish Miecting.-Lunacy Acts in New South Wales.Obituary.-Appoinfments.-Index Medico-Paychologicus, \&c. 


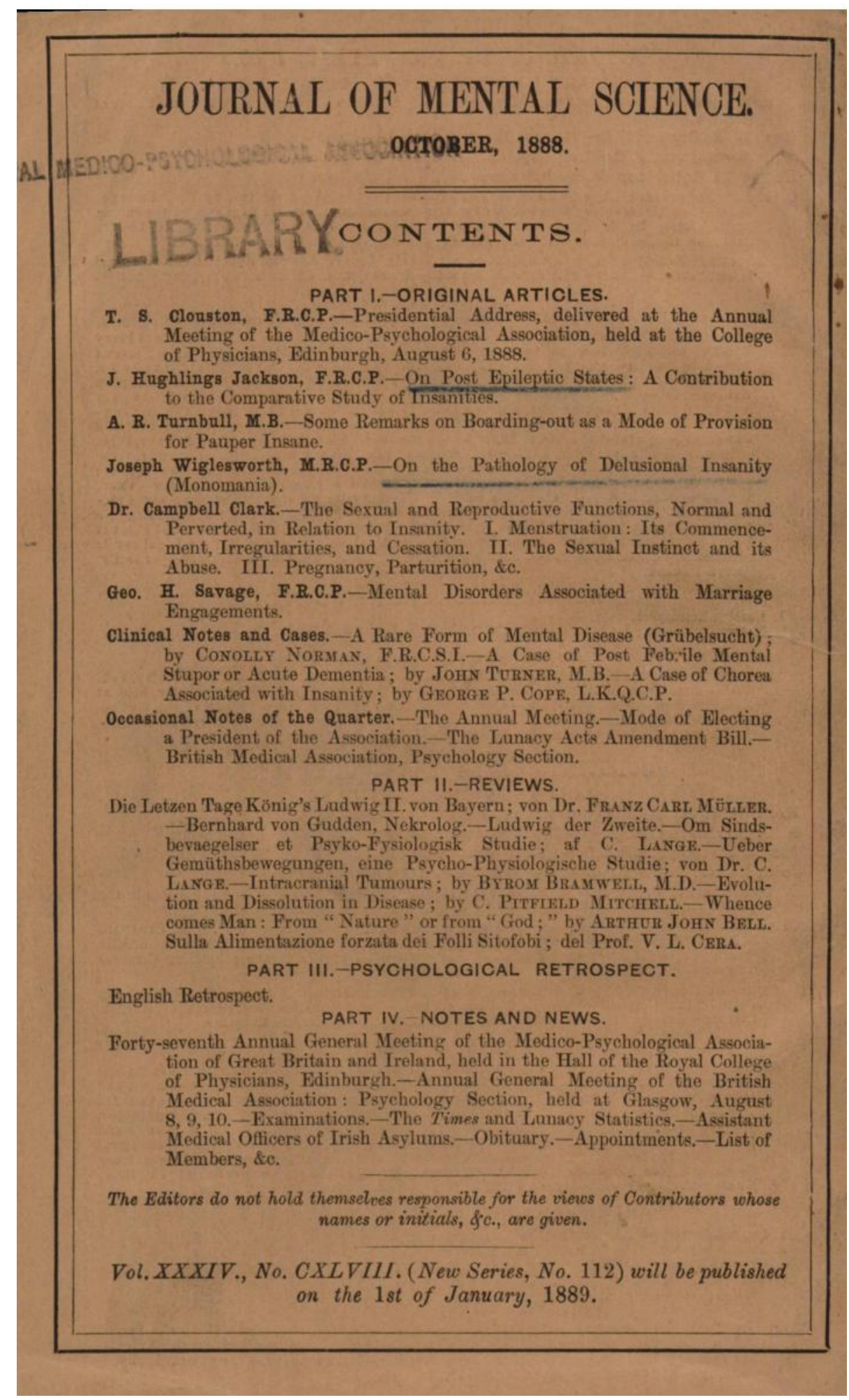

